\title{
Seasonal variation of bioactive properties and phenolic composition of Cynara cardunculus var. altilis
}

\author{
Filipa Mandim $^{\mathrm{a}, \mathrm{b}}$, Spyridon A. Petropoulos ${ }^{\mathrm{c}}$, Kyriakos D. Giannoulis ${ }^{\mathrm{c}}$, Maria Inês Dias ${ }^{\mathrm{a}}$, \\ Ângela Fernandes ${ }^{\mathrm{a}}$, José Pinela ${ }^{\mathrm{a}}$, Marina Kostic ${ }^{\mathrm{d}}$, Marina Sokovićc ${ }^{\mathrm{d}}$, Lillian Barros ${ }^{\mathrm{a}, *}$, \\ Celestino Santos-Buelga ${ }^{\mathrm{b}}$, Isabel C.F.R. Ferreira ${ }^{\mathrm{a}, *}$ \\ ${ }^{a}$ Centro de Investigação de Montanha (CIMO), Instituto Politécnico de Bragança, Campus de Santa Apolónia, 5300-253 Bragança, Portugal \\ ${ }^{\mathrm{b}}$ Grupo de Investigación en Polifenoles (GIP-USAL), Facultad de Farmacia, Universidad de Salamanca, Campus Miguel de Unamuno s/n, 37007 Salamanca, Spain \\ ${ }^{\mathrm{c}}$ University of Thessaly, Department of Agriculture, Crop Production and Rural Environment, 38446 N. Ionia, Volos, Greece \\ ${ }^{\mathrm{d}}$ Institute for Biological Research "Siniša Stanković"- National Institute of Republic of Serbia, University of Belgrade, Bulevar despota Stefana 142, Belgrade, Serbia
}

\section{A R T I C L E I N F O}

\section{Keywords:}

Anti-inflammatory activity

Antimicrobial properties

Bioactive properties

Cynara cardunculus (L.)

Phenolic compounds

Seasonal variation

\begin{abstract}
A B S T R A C T
Cynara cardunculus L. (cardoon) has several health benefits mainly attributed to its abundance in polyphenols. In this study, cardoon heads (capitula) were harvested in Greece during the flowering stage, and the hydroethanolic extracts were assessed in terms of phenolic compounds composition and antioxidant, cytotoxic, anti-inflammatory, and antimicrobial activities. The phenolic profile was evaluated by HPLC-DAD-ESI/MS to better understand the seasonal changes in the individual compound levels and how these changes correlate with bioactive properties. The main phenolic compounds identified were caffeoylquinic and dicaffeoylquinic acid derivatives. Immature heads (Car A: principal growth stage (PGS) 5) had the highest phenolic content (34.3 mg/ g) and cytotoxic $\left(\mathrm{GI}_{50}\right.$ of $69-268 \mu \mathrm{g} / \mathrm{mL}$ ) and anti-inflammatory ( $\mathrm{IC}_{50}$ of $183 \mu \mathrm{g} / \mathrm{mL}$ ) activities. Sample Car D (PGS 6/7) revealed the highest antioxidant (IC ${ }_{50}$ of $23-227 \mu \mathrm{g} / \mathrm{mL}$ ) and antifungal (MIC of $0.26-0.51 \mathrm{mg} / \mathrm{mL}$ ) potential. Regarding the antibacterial activity, Car E (PGS 7) revealed the best results (MIC of 0.59-1.18 mg/ $\mathrm{mL}$ ). This study suggests that the maturity stage of the plant influences the phenolic composition and bioactivity.
\end{abstract}

\section{Introduction}

Cynara cardunculus L. (syn. cardoon) is a diploid $(2 n=34)$ crosspollinated perennial plant that belongs to the Asteraceae (sunflower) family. This species includes three taxa: the cultivated cardoon $(C$. cardunculus var. altilis DC.), the wild cardoon (C. cardunculus var. sylvestris), and their ancestor globe artichoke (C. cardunculus var. scolymus) (Conceição et al., 2016).

Indigenous to the Mediterranean region, this crop is an integral constituent of the so-called "Mediterranean diet", used since antique times in dishes such as salads and soups. Its inflorescences are also widely used in the manufacturing of protected designation of origin (PDO) cheeses, as a result of the clotting capacity. The oil from cardoon seeds is used in the biodiesel production and as edible vegetable oil, while fresh and dried plants for biomass production (Conceição et al., 2016; Gominho, Curt, Lourenço, Fernández, \& Pereira, 2018; Piluzza, Molinu, Re, \& Sulas, 2019). Besides that, this crop is widely consumed as herbal medicine, due to the known health-promoting effects. Several studies have demonstrated its anticarcinogenic, antioxidant, antimicrobial, anti-inflammatory, anti-HIV, bile-expelling, hepatoprotective, and hypocholesterolemic potential (Gostin \& Waisundara, 2019; Kollia, Markaki, Zoumpoulakis, \& Proestos, 2017; Petropoulos, Fernandes, Pereira, 2019; Petropoulos, Fernandes, Tzortzakis, et al., 2019; Scavo et al., 2019). Moreover, recently the commercial and economic potential of cardoon has been recognized due to its nutritional value, bioactive potential, as well as its diverse industrial applications. Parameters such as the high crop yield, the stability of agronomic performance, the adaptation capacity to climate changes and the resistance to different types of abiotic stress, are important contributors to the stimulation of this interest (Conceição et al., 2016; Gominho et al., 2018). The pharmaceutical and nutritional properties associated with this crop could be correlated to compounds with beneficial effects, namely flavonoids (apigenin and luteolin derivatives), phenolic acids (mainly caffeoylquinic and dicaffeoylquinic acid derivatives), and anthocyanins (cyanidin), found in different plant tissues of cardoon, in addition to inulin, fibers and minerals (Petropoulos, Pereira, Ntatsi, et al., 2018).

The existence of phenolic compounds in different plant species has

\footnotetext{
* Corresponding authors.

E-mail addresses: lillian@ipb.pt (L. Barros), iferreira@ipb.pt (I.C.F.R. Ferreira).
} 
November 2017 - October 2018

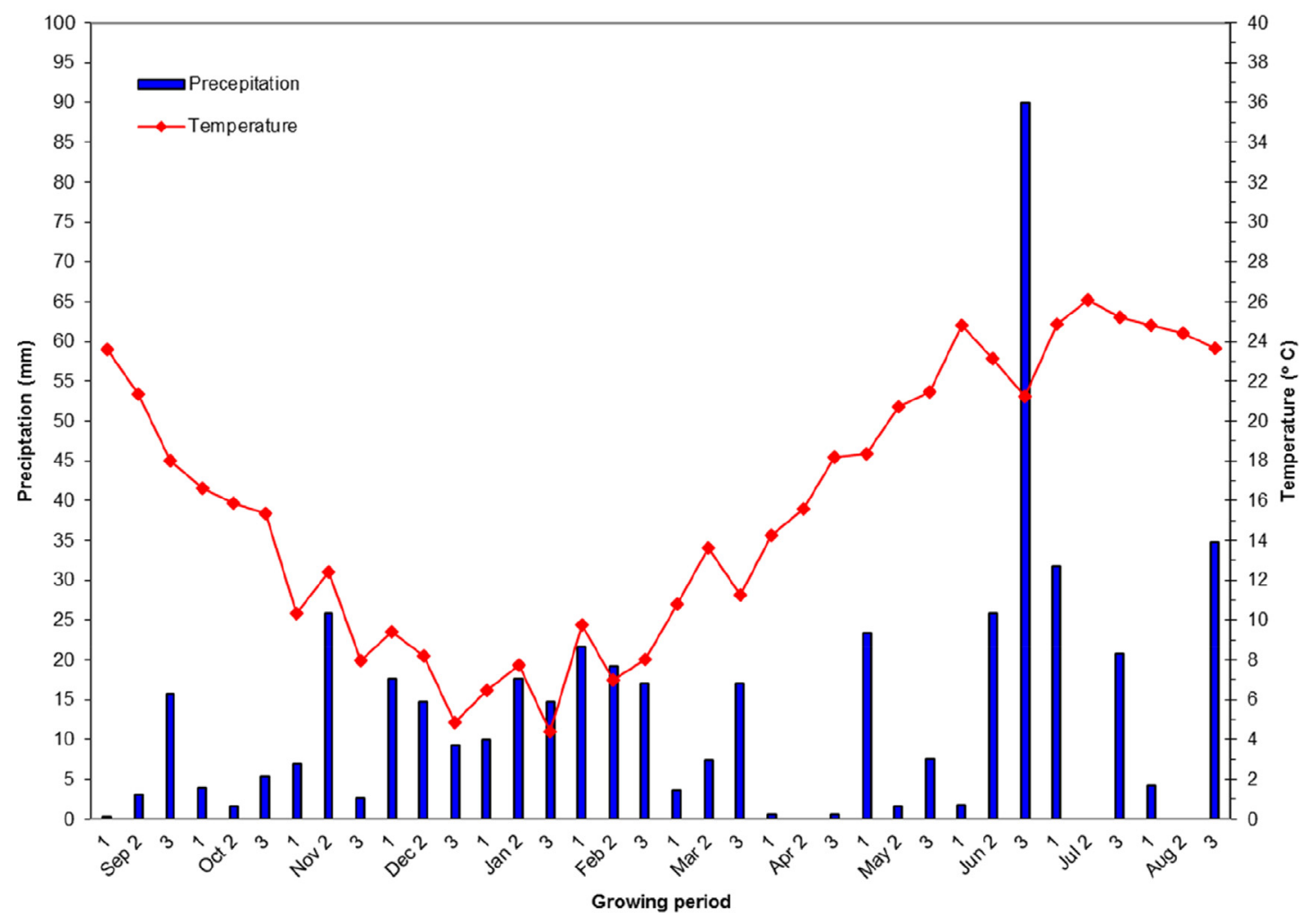

Fig. 1. Meteorological data (mean temperature and precipitation) during the 2017-2018 growing period. Each month is separated in three 10-day intervals (1, 2 and 3).

been extensively studied due to the high variety and potential shown. Their presence and abundance are related to metabolic reactions which are strongly influenced by parameters such as the vegetable tissue analyzed, the physiological (growth) age of the species, the different stages of development (harvesting date), the environmental conditions and interactions, the cultivation practices, and after-harvest conditions (genetic background and geographical location) (Ferreira, Martins, \& Barros, 2017). The presence of caffeic acid derivatives, namely chlorogenic acid and cynarine (i.e., 1,5-dicaffeoylquinic acid), in cardoon parts is referred by several authors who suggest the pivotal role of these compounds to the pharmaceutical and nutritional properties associated with this crop (Russo et al., 2017). Therefore, it is of great importance from the production, economical and industrial point of view, to reveal the chemical composition and biological potential of different plant parts through the growing season. Available information suggests that the qualitative and quantitative phenolic composition, and also the biological potential appears to be strongly influenced by parameters such as the environmental conditions, and the genetic background. The full description of the polyphenolic profile and bioactivities of cardoon different parts as affected by factors such as the maturity stage, the genotype or the growing locations, is very important to validate all of its applications and could contribute to the management of agricultural by-products, for the sustainable agricultural practices and the circular economy of the crop (Dias et al., 2018; Pandino, Lombardo, Williamson, \& Mauromicale, 2012; Petropoulos, Fernandes, Pereira, et al., 2019; Petropoulos, Fernandes, Tzortzakis, et al., 2019).

Therefore, the present study aimed to evaluate the variations in the phenolic composition and bioactive potential of cardoon heads harvested during the flowering period. According to the findings, the main goal is to provide integrated knowledge towards the improved use of cardoon, regarding the associated potential and the optimal time of the year to harvest its heads and further maximize their added value and their contribution to human health.

\section{Materials and methods}

\subsection{Standards and reagents}

The solvents used were of analytical grade and were obtained from Fisher Scientific (Lisbon, Portugal). The sulphorhodamine B (SRB), Trolox (6-hydroxy-2,5,7,8-tetramethylchroman-2-carboxylic acid), AAPH (2,2'-azobis(2-methylpropionamide) dihydrochloride), ellipticine, tocol, trypan blue, and Tris (tris-(hydroxymethyl) aminomethane) were acquired from Sigma-Aldrich (St. Louis, MO, USA). The phenolic compounds' standards were acquired from Extrasynthèse (Genay, France). The RPMI-1640 and DMEM mediums, foetal bovine serum (FBS), L-glutamine, Hank's balanced salt solution (HBSS), trypsin-EDTA (ethylenediaminetetraacetic acid), and penicillin/streptomycin solution (100 U/mL and $100 \mathrm{mg} / \mathrm{mL}$, respectively) were obtained from Hyclone (Logan, Utah, USA). From Biomerieux (Marcy l'Etoile, France) was obtained the Tryptic Soy Broth (TSB). Blood agar with 7\% sheep blood and MacConkey agar plates were purchased from Liofilchem (Italy). Other reagents and solvents of analytical grade were obtained from common sources. Water was treated with a Milli-Q water purification system (TGI Pure Water Systems, Greenville, SC, USA).

\subsection{Plant material}

The heads (capitula) of Cynara cardunculus var. altilis DC. cv. Bianco Avorio (Fratelli Ingegnoli Spa, Milano, Italy) were harvested in central Greece at the experimental field of the University of Thessaly in Velestino (22.756E, $39.396 \mathrm{~N}$ ), during the growing period of 2017-2018 (principal growth stages (PGS) 5-8, according to the Biologische Bundesanstalt, Bundessortenamt, CHemische Industrie (BBCH) scale) (Archontoulis, Struik, Vos, \& Danalatos, 2013). The samples were collected at the beginning of the flower's development, during full maturity, and in seed ripening stages from the main 
inflorescence of each plant. For each harvesting date, 15 individual primary heads $(n=15)$ were collected from different plants. Six different samples of cardoon were analyzed corresponding to six harvesting dates, namely Car A collected at the end of April (PGS 5), Car B collected at the beginning of May (PGS 5/6), Car C collected at the end of May (PGS 6), Car D collected in June (PGS 6/7), and finally the samples Car E and Car F collected at the beginning (PGS 7) and at the end of August (PGS 8), respectively. The climate conditions during the growing period of 2017-2018 are presented in Fig. 1.

The plant material was freeze-dried (Sublimator model EKS, Christian Zirbus Co., Germany), ground to a fine powder ( $\sim 20$ mesh) using a domestic electric blender, and mixed to obtain homogenate batch samples. Then, the samples were put in air-sealed bags and stored under deep freezing conditions $\left(-80{ }^{\circ} \mathrm{C}\right)$ under protection from light until further analysis.

\subsection{Extraction procedure}

To obtain the extracts, each sample $(1.5 \mathrm{~g})$ was stirred $(150 \mathrm{rpm})$ at room temperature for $1 \mathrm{~h}$ with $\mathrm{EtOH} / \mathrm{H}_{2} \mathrm{O}(80: 20, v / v ; 30 \mathrm{~mL}$ ) (Bessada, Barreira, Barros, Ferreira, \& Oliveira, 2016). The mixture obtained was filtered through a Whatman No. 4 paper and evaporated under reduced pressure (rotary evaporator Hei-VAP Advantage, Heidolph, Germany) at $40{ }^{\circ} \mathrm{C}$. The aqueous phase was frozen at $-20{ }^{\circ} \mathrm{C}$ and lyophilized (FreeZone 4.5, Labconco, Kansas City, MO, USA) before carrying out the remaining analyses.

\subsection{Phenolic composition analysis}

The final lyophilized extracts were re-dissolved in EtOH/ $\mathrm{H}_{2} \mathrm{O}$ $(20: 80, v / v)$ to prepare solutions at $10 \mathrm{mg} / \mathrm{mL}$. Then, the obtained solutions were filtered through $0.22 \mu \mathrm{m}$ nylon syringe filters. The polyphenolic composition was determined by HPLC coupled to a diode array detector and electrospray ionization - mass spectrometry (HPLCDAD-ESI/MS), according to the conditions previously described by Bessada et al. (2016). The tentative identification of the phenolic compounds was based on the comparison of the retention times, and the UV-Vis spectra obtained from commercial standards and the literature information. For the quantification, the area of the peaks was determined and then compared with the calibration curves of the most similar available commercial standards. The results were expressed as $\mathrm{mg}$ per $\mathrm{g}$ of dry weight of extract.

\subsection{Evaluation of the bioactive properties}

\subsubsection{Antioxidant activity}

Two cell-based in vitro methodologies were used for the measurement of the antioxidant capacity: the inhibition of lipid peroxidation by the decrease in thiobarbituric acid reactive substances (TBARS), and the oxidative hemolysis inhibition assay (OxHLIA). The positive control used was the commercial antioxidant Trolox.

TBARS assay. The inhibition capacity of the tested samples against the formation of TBARS, such as malondialdehyde generate from the $e x$ vivo decomposition of lipid peroxidation products, was evaluated using porcine brain (Sus scrofa) cell homogenates $(1: 2, w / v ; 0.1 \mathrm{~mL}$ ), according to the methodology previously described by Souilem et al. (2017). The extracts obtained (see Section 2.3) were re-dissolved in $\mathrm{H}_{2} \mathrm{O}$, obtaining solutions with a final concentration of $5 \mathrm{mg} / \mathrm{mL}$, while further successive dilutions led to obtaining the range of the concentrations tested (2500 to $10 \mu \mathrm{g} / \mathrm{mL}$ ). The results obtained were expressed as $\mathrm{IC}_{50}$ values $(\mu \mathrm{g} / \mathrm{mL})$, corresponding to the sample concentration that provides $50 \%$ of antioxidant activity.

OxHLIA assay. The antihemolytic activity of the extracts was determined following the methodology previously described by Mandim et al. (2019). The erythrocytes used in the assay were isolated from sheep blood samples collected from healthy animals. In a 48-well plate, the erythrocyte solution ( $2.8 \%$ in PBS, $v / v ; 200 \mu \mathrm{L}$ ) was mixed with $400 \mu \mathrm{L}$ of either PBS solution (control), antioxidant sample dissolved in PBS, or water (for complete hemolysis). After pre-incubating the mixture at $37{ }^{\circ} \mathrm{C}$ for $10 \mathrm{~min}$ with shaking, AAPH (160 mM in PBS; $\left.200 \mu \mathrm{L}\right)$ was added and the optical density was measured at $690 \mathrm{~nm}$. Then, the plate was incubated under the same conditions and the optical density was measured each $10 \mathrm{~min}$ at the same wavelength until complete hemolysis occurred. The results were calculated as explained by Mandim et al. (2019) and expressed as $\mathrm{IC}_{50}$ values $(\mu \mathrm{g} / \mathrm{mL})$ at $\Delta t 60$ and $120 \mathrm{~min}$, which correspond to the extract concentration required to keep $50 \%$ of the erythrocyte population intact for 60 and $120 \mathrm{~min}$, respectively.

\subsubsection{Anti-inflammatory activity}

The anti-inflammatory activity was determined according to the method formerly reported by Mandim et al. (2019). The nitrite concentration in the culture medium was determined to assess the capacity of the extracts to inhibit the lipopolysaccharide (LPS)-induced NO production through a murine macrophage cell line (RAW 264.7). Cardoon heads extracts (see Section 2.3) re-dissolved in water at a final concentration of $8 \mathrm{mg} / \mathrm{mL}$ were tested. The positive control used was Dexamethasone, and samples without LPS were used as negative control. The results were expresses as $\mathrm{IC}_{50}$ values $(\mu \mathrm{g} / \mathrm{mL})$, which correspond to the extract concentration responsible for $50 \%$ of NO production inhibition.

\subsubsection{Cytotoxic and hepatotoxic potential}

The cardoon head extracts prepared in Section 2.3 were re-dissolved in water at a concentration of $8 \mathrm{mg} / \mathrm{mL}$ and subjected to successive dilutions to obtain the concentrations to be tested $(6.25-400 \mu \mathrm{g} / \mathrm{mL})$. Four human tumor cell lines (HeLa - cervical carcinoma; HepG2 - hepatocellular carcinoma; MCF-7 - breast carcinoma; and NCI-H460 non-small cell lung cancer) (all acquired from Leibniz-Institut DSMZ), and a non-tumor porcine liver primary culture (PLP2) were tested. The cytotoxic and hepatotoxic activities were tested through the sulforhodamine B colorimetric test, as described by Barros et al. (2013). Ellipticine was used as a positive control. The results were expressed as $\mathrm{GI}_{50}$ values $(\mu \mathrm{g} / \mathrm{mL})$, which translate the extract concentration responsible for $50 \%$ of inhibition of cell proliferation.

\subsubsection{Antimicrobial activity}

The obtained extracts were re-dissolved in 5\% DMSO and diluted according to the protocol described by Petropoulos, Fernandes, Pereira, et al. (2019), Petropoulos, Fernandes, Tzortzakis, et al. (2019). The following Gram-positive bacteria: Bacillus cereus (food isolate), Staphylococcus aureus (ATCC 6538) and Listeria monocytogenes (NCTC 7973); and Gram-negative bacteria: Escherichia coli (ATCC 35210), Enterobacter cloacae (human isolate), Salmonella typhimurium (ATCC 13311) were tested. The antifungal assay was performed with the micromycetes Aspergillus fumigatus (ATCC 1022), A. versicolor (ATCC 11730), A. niger (ATCC 6275), Penicillium ochrochloron (ATCC 9112), P. funiculosum (ATCC 36839) and $P$. aurantiogriseum ( $P$. verrucosum var. cyclopium (food isolate)), following the methodology previously described by Petropoulos, Fernandes, Pereira, et al. (2019), Petropoulos, Fernandes, Tzortzakis, et al. (2019). The microorganisms are deposited at Mycological laboratory, Department of Plant Physiology, Institute for biological research "Sinisa Stanković", University of Belgrade, Serbia. Streptomycin, ampicillin, ketoconazole, and bifonazole were used as positive controls. The results were expressed as minimum inhibitory (MIC) and minimum bactericidal (MBC) or fungicidal (MFC) concentrations $(\mathrm{mg} / \mathrm{mL})$.

\subsection{Statistical analysis}

The performed experiments were carried out in triplicate, and the results are expressed as mean \pm standard deviation. Means and 
Table 1

Phenolic compounds composition of the hydroethanolic extracts of Cynara cardunculus var. altilis heads. It is presented the retention time (Rt), wavelengths of maximum absorption $\left(\lambda_{\max }\right)$ in the UV-Vis region, mass spectral data and tentative identification of phenolic compounds.

\begin{tabular}{|c|c|c|c|c|c|}
\hline Peak & Rt (min) & $\lambda_{\max }(\mathrm{nm})$ & {$[\mathrm{M}-\mathrm{H}]^{-}(m / z)$} & $\operatorname{MS}^{2}(m / z)$ & Tentative identification \\
\hline 1 & 7.00 & 325 & 353 & 191(100), 179(10), 161(5), 135(5) & 5-O-Caffeoylquinic acid \\
\hline 2 & 12.08 & 310 & 325 & $163(100), 145(5), 119(30)$ & $p$-Coumaric acid hexoside \\
\hline 3 & 13.34 & 325 & 607 & $445(100), 269(47)$ & Apigenin- $O$-glucuronide- $O$-hexoside \\
\hline 4 & 16.77 & 319 & 677 & 515(100), 353(9), 191(3) & Tri-O-caffeoylquinic acid \\
\hline 5 & 18.68 & 345 & 461 & $285(100)$ & Luteolin-7-O-glucuronide \\
\hline 6 & 18.84 & 345 & 461 & $285(100)$ & Luteolin-O-glucuronide \\
\hline 7 & 20.48 & 344 & 515 & $353(100), 191(5), 173(5), 161(5), 135(5)$ & 3,5-O-Dicaffeoylquinic acid \\
\hline 8 & 21.52 & 332 & 577 & $269(100)$ & Apigenin-7-O-rutinoside \\
\hline 9 & 22.93 & 336 & 445 & $269(100)$ & Apigenin-7-O-glucuronide \\
\hline 10 & 28.10 & 332 & 517 & 473(100), 269(50) & Apigenin-O-malonylhexoside \\
\hline
\end{tabular}

standard deviations were determined from the obtained data using Microsoft Excel. All statistical tests were performed using SPSS Statistics software (IBM SPSS Statistics for Windows, Version 22.0. Armonk, NY: IBM Corp.). The results were obtained through the analysis of variance (ANOVA), and by Tukey's HSD test ( $\alpha=0.05)$.

\section{Results and discussion}

\subsection{Composition in phenolic compounds}

The detected phenolic compounds of $C$. cardunculus heads and their quantification (mg/g extract) are presented in Tables 1 and 2, respectively. The phenolic compounds were tentatively identified according to their retention time (Rt), the wavelength of maximum absorbance $\left(\lambda_{\max }\right)$, pseudomolecular ion $\left([\mathrm{M}-\mathrm{H}]^{-}\right)$, and fragmentation pattern $\left(\mathrm{MS}^{2}\right)$. Ten phenolic compounds were found, namely four phenolic acid derivatives (peaks 1, 2, 4 and 7) and six flavone glycosides (3, 5, 6, 8, 9 and 10), the latter being present in higher concentrations. Fig. 2 shows the HPLC phenolic profile of the hydroethanolic extract of the sample Car A. A significant number of previous publications have already given a thorough description of the phenolic profile of cardoon flower and its constituents, and hence the tentative identification of the peaks $1,5,7$, 8, and 9 (5-O-caffeoylquinic acid, luteolin-7-O-glucuronide, 3,5-O-dicaffeoylquinic acid, apigenin-7-O-rutinoside, and apigenin-7-O-glucuronide, respectively) has already been described by the authors in the study performed by Dias et al. (2018) in inflorescences of cardoon samples originated from Portugal. For peaks 4 and 6 (tri-O-caffeoylquinic acid and luteolin-O-glucuronide), the tentative identification was based on the previously described by Gouveia and Castilho (2012) in artichoke juice and capsules, respectively. Peak 2 was tentatively identified as $p$-coumaric acid hexoside, as previously described in globe artichoke leaf blades by Petropoulos, Pereira, Barros, and Ferreira (2017). Peak 3 was tentatively identified as apigenin-O-glucuronide- $O$ hexoside based on the previous description made by Pereira, Barros, Carvalho, Santos-Buelga, and Ferreira (2015) in hydroethanolic extracts and infusions of flowering stems and capitula of artichoke. Finally, peak 10 (apigenin- $O$-malonylhexoside) was tentatively identified based on the description made by Pandino et al. (2012) in cultivated cardoon and globe artichoke samples from Italy.

Quantitatively, cardoon heads in the earliest maturation stage (Car A) presented the highest content in phenolic compounds, mainly due to the presence of apigenin-7-O-rutinoside and 5-O-caffeoylquinic acid. Overall, the compounds present in higher quantities in all studied samples were 5-O-caffeoylquinic acid $(0.445-3.55 \mathrm{mg} / \mathrm{g}$ extract) and apigenin-7-O-rutinoside $(0.97-3.51 \mathrm{mg} / \mathrm{g}$ extract). It has been verified that the maturity stage shows a significant influence, not only on the content of total phenolic compounds, but also on the polyphenolic profile, while environmental conditions during harvesting may also impose a significant impact on the phenolic compounds composition. According to Lombardo et al. (2010), a significant variation in phenolic compounds composition in inflorescences of globe artichoke was observed depending on the harvesting time (winter or spring harvest), with several polyphenols being detected only in one of the two harvests. In the study of Pandino, Lombardo, Lo Monaco, and Mauromicale (2013), an increase of phenolic compounds content in receptacles was observed from February to April followed by a concomitant decrease in the other plant parts. This finding could suggest a translocation of phenolic compounds to the most biosynthetically active plant parts.

Table 2

Phenolic compounds quantification in the hydroethanolic extracts of Cynara cardunculus var. altilis heads with different maturation stages.

\begin{tabular}{|c|c|c|c|c|c|c|c|}
\hline \multirow[t]{2}{*}{ Peak } & \multirow[t]{2}{*}{ Tentative identification } & \multicolumn{6}{|c|}{ Content (mg/g extract) } \\
\hline & & Car A & Car B & Car C & Car D & Car E & Car F \\
\hline 1 & 5-O-Caffeoylquinic acid & $2.09 \pm 0.01^{\mathrm{c}}$ & $2.51 \pm 0.01^{\mathrm{b}}$ & $3.55 \pm 0.02^{\mathrm{a}}$ & $0.445 \pm 0.001^{\mathrm{e}}$ & n.d. & n.d. \\
\hline 2 & $p$-Coumaric acid hexoside & $1.40 \pm 0.02^{\mathrm{a}}$ & $0.82 \pm 0.02^{\mathrm{c}}$ & $0.40 \pm 0.01^{\mathrm{d}}$ & $0.225 \pm 0.001^{\mathrm{e}}$ & n.d. & n.d. \\
\hline 3 & Apigenin- $O$-glucuronide- $O$-hexoside & $0.636 \pm 0.001^{\mathrm{c}}$ & $1.07 \pm 0.02^{\mathrm{a}}$ & $0.960 \pm 0.004^{\mathrm{b}}$ & $0.514 \pm 0.004^{\mathrm{d}}$ & n.d. & n.d. \\
\hline 4 & Tri-O-caffeoylquinic acid & $1.29 \pm 0.02^{\mathrm{a}}$ & $0.97 \pm 0.01^{\mathrm{b}}$ & $0.88 \pm 0.03^{\mathrm{c}}$ & $0.749 \pm 0.004^{\mathrm{d}}$ & n.d. & n.d. \\
\hline 5 & Luteolin-7-O-glucuronide & $0.775 \pm 0.003^{\mathrm{a}}$ & $0.90 \pm 0.02^{\mathrm{c}}$ & $0.60 \pm 0.01^{\mathrm{d}}$ & $0.60 \pm 0.01^{\mathrm{d}}$ & n.d. & n.d. \\
\hline 6 & Luteolin-O-glucuronide & $0.564 \pm 0.001^{\mathrm{c}}$ & $1.03 \pm 0.01^{\mathrm{a}}$ & $0.517 \pm 0.001^{\mathrm{e}}$ & $0.563 \pm 0.001^{\mathrm{d}}$ & n.d. & n.d. \\
\hline 7 & 3,5-O-Dicaffeoylquinic acid & $9.9 \pm 0.2^{\mathrm{a}}$ & $8.0 \pm 0.2^{\mathrm{b}}$ & $3.81 \pm 0.03^{\mathrm{c}}$ & $2.81 \pm 0.03^{\mathrm{e}}$ & $0.65 \pm 0.01^{\mathrm{f}}$ & $0.407 \pm 0.002^{g}$ \\
\hline 8 & Apigenin-7-O-rutinoside & $3.17 \pm 0.01^{\mathrm{b}}$ & $3.51 \pm 0.02^{\mathrm{a}}$ & $1.416 \pm 0.002^{\mathrm{e}}$ & $0.97 \pm 0.04^{\mathrm{f}}$ & $2.22 \pm 0.03^{c}$ & $1.60 \pm 0.01^{\mathrm{d}}$ \\
\hline 9 & Apigenin-7-O-glucuronide & $13.2 \pm 0.2^{\mathrm{a}}$ & $8.96 \pm 0.04^{\mathrm{c}}$ & $3.04 \pm 0.01^{\mathrm{d}}$ & $1.261 \pm 0.003^{\mathrm{e}}$ & n.d. & n.d. \\
\hline \multirow[t]{4}{*}{10} & Apigenin- $O$-malonylhexoside & $1.170 \pm 0.005^{\mathrm{a}}$ & $1.002 \pm 0.002^{\mathrm{b}}$ & $0.72 \pm 0.01^{c}$ & $0.70 \pm 0.03^{\mathrm{d}}$ & n.d. & n.d. \\
\hline & Total phenolic acids & $14.7 \pm 0.2^{\mathrm{a}}$ & $12.3 \pm 0.2^{\mathrm{b}}$ & $8.64 \pm 0.09^{c}$ & $4.23 \pm 0.02^{\mathrm{d}}$ & $0.65 \pm 0.01^{\mathrm{e}}$ & $0.407 \pm 0.002^{\mathrm{f}}$ \\
\hline & Total flavonoids & $19.5 \pm 0.2^{\mathrm{a}}$ & $16.48 \pm 0.04^{\mathrm{b}}$ & $7.26 \pm 0.02^{\mathrm{c}}$ & $4.608 \pm 0.01^{\mathrm{d}}$ & $2.22 \pm 0.03^{\mathrm{e}}$ & $1.60 \pm 0.01^{\mathrm{f}}$ \\
\hline & Total phenolic compounds & $34.3 \pm 0.4^{\mathrm{a}}$ & $28.8 \pm 0.2^{\mathrm{b}}$ & $15.89 \pm 0.07^{\mathrm{c}}$ & $8.84 \pm 0.02^{\mathrm{d}}$ & $2.87 \pm 0.04^{\mathrm{e}}$ & $2.01 \pm 0.01^{\mathrm{f}}$ \\
\hline
\end{tabular}

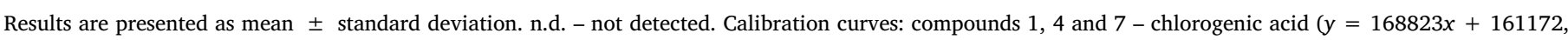

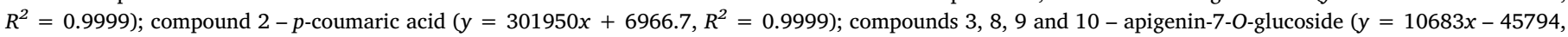

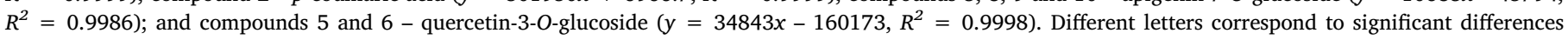
$(p<0.05)$. Mean statistical differences obtained by Student's $t$-test. 


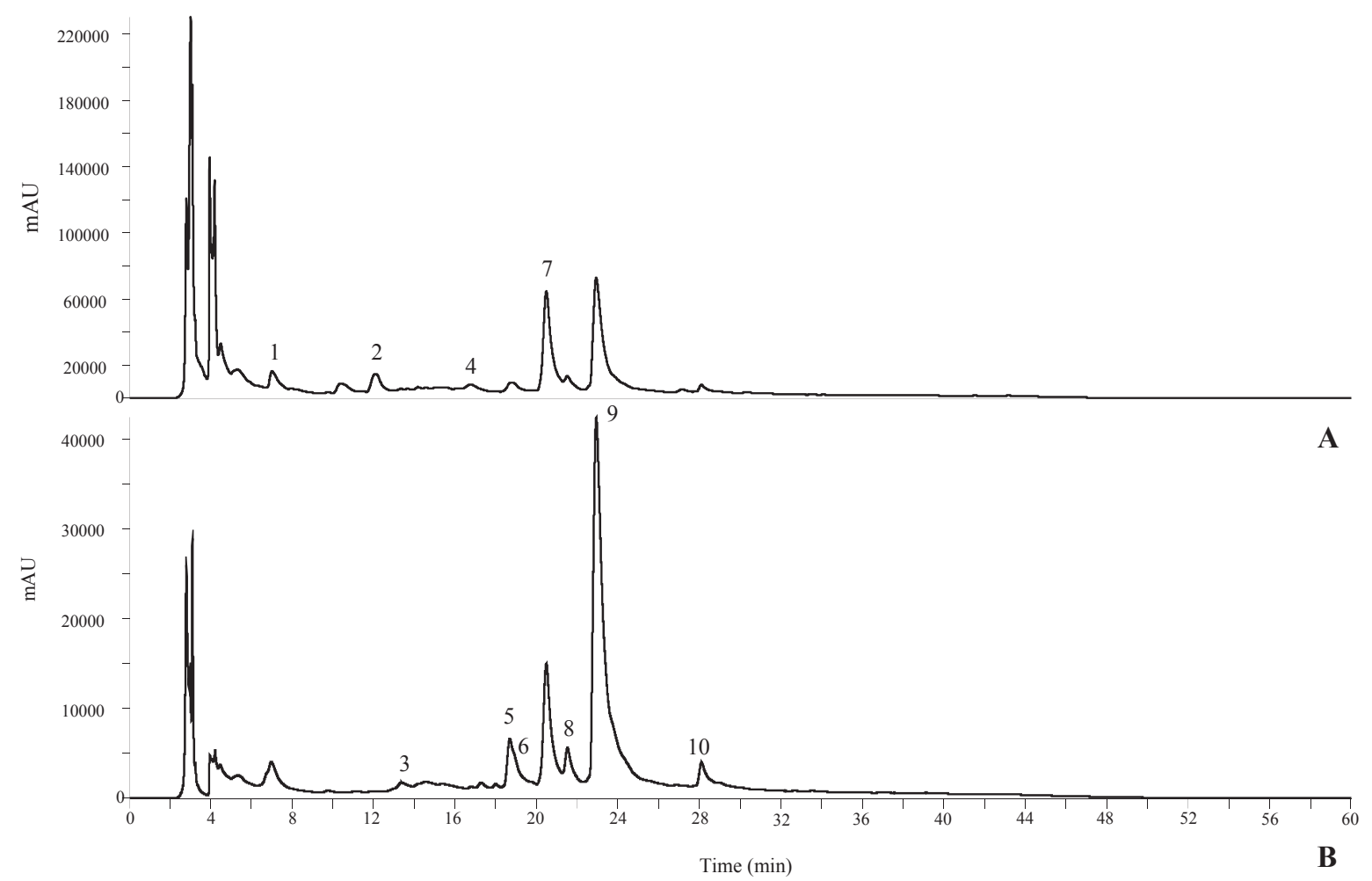

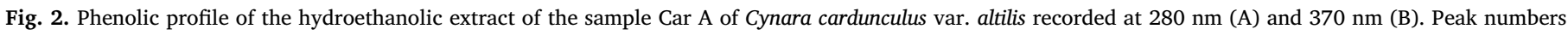
correspond to the compounds identified in Table 1.

Similar trends were observed in our study, where the phenolic compounds content decreased with maturity progress, suggesting a possible translocation of polyphenols to rhizomes which act as reserves for the next vegetative period (Alghazeer et al., 2012). Studies with cardoon leaves suggest that the content in bioactive compounds such as carbohydrates, flavonoids, and other polyphenols increases progressively with the age of the plant (Wahba, Sarhan, Salama, Sharaf-Eldin, \& Gad, 2017). Also, different cultivars, genotypes, growing conditions, and plant parts present a considerable variation source regarding the phenolic compounds content. According to Pandino et al. (2012), total phenolic compounds in outer and inner bracts and receptacle varies among various cardoon genotypes and plant parts. The same authors also found that the cultivars and plant parts may affect the content of phenolic compounds. In the study of Pandino, Courts, Lombardo, Mauromicale, and Williamson (2010), apigenin derivatives were detected in the highest concentrations in both wild and cultivated cardoon immature inflorescences. Ramos et al. (2014) studied the polyphenolic composition of different plant tissues of cardoon cultivated in the south of Portugal and found different phenolic profiles depending on the plant tissue under analysis, which were characterized by high levels of hydroxycinnamic acids, mainly dicaffeoylquinic acids in receptacle and bracts, and apigenin and luteolin derivatives in capitulum florets and leaves, respectively. Dias et al. (2018), who analyzed the phenolic profile and bioactivity of inflorescences of different cardoon genotypes, found that the genotype 'F1-25-4' stands out as the one with the highest content of phenolic compounds, being the best option for protected designation of origin (PDO) cheeses production.

Similar to our results, previous reports on the composition of phenolic compounds suggest that cardoon parts have a higher flavonoid content compared to phenolic acid derivatives (Pandino et al., 2012; Petropoulos et al., 2017). Particularly for head parts, Lombardo et al. (2010) observed significant differences in the phenolic compounds profile of spring- and winter-harvested globe artichoke heads, with results in accordance with the present study, where the majority of the tested samples had higher abundance in flavonoids than in hydroxycinnamoyl derivatives, except the sample Car C. Moreover, the polyphenolic profile of the tested cardoon heads was in general consistent with the literature information (Dias et al., 2018; Petropoulos, Pereira, Tzortzakis, et al., 2018).

\subsection{Bioactive properties}

The results regarding the cell-based antioxidant activity are presented in Table 3. Amongst the harvesting stages analyzed, sample Car $\mathrm{D}$ revealed the lowest $\mathrm{IC}_{50}$ values for the TBARS assay, while a varied response of the tested samples was observed for the OxHLIA test. In particular, samples Car A and D were the most effective (lowest $\mathrm{IC}_{50}$ values) in hemolysis delay at $60 \mathrm{~min}\left(\Delta t_{60}\right)$ and samples Car $\mathrm{A}, \mathrm{D}$, and $\mathrm{F}$ at $120 \mathrm{~min}\left(\Delta t_{120}\right)$. Nevertheless, these $\mathrm{IC}_{50}$ values are higher than those of the Trolox, used as a positive control. However, it should be considered that Trolox is a standard antioxidant compound, and the extracts tested are a complex mixture with phytochemicals that may not have antioxidant potential or show antagonistic effects among them. The extract obtained at principal growth stage (PGS) 7 (Car E) revealed the lower effectiveness in the inhibition of reactive substances formation generated by the ex vivo decomposition of lipid peroxidation products and did not reveal potential in protecting the erythrocyte population from the haemolytic action. Moreover, all the tested extracts revealed lower capacity in inhibiting the formation of malondialdehyde and other reactive substances that are generated from the ex vivo decomposition of lipid peroxidation products.

The antioxidant potential of cardoon plant tissues is widely described in the literature, mainly through the free radical scavenging capacity, measured by the DPPH assay. Several in vitro assays with different parts of cardoon plants highlight the antioxidant potential of this crop (Petropoulos et al., 2018Petropoulos, Pereira, Ntatsi, et al., 2018; Petropoulos, Pereira, Tzortzakis, et al., 2018; Piluzza et al., 2019). Furthermore, it has been reported that the antioxidant potential differed significantly among the different plant parts and/or the various genotypes tested, including the genotype evaluated in this study 
Table 3

Antioxidant, cytotoxic and anti-inflammatory activities of Cynara cardunculus var. altilis head extracts.

\begin{tabular}{|c|c|c|c|c|c|c|c|}
\hline & Car A & Car B & Car C & Car D & Car E & Car F & Positive control \\
\hline Antioxidant activity $\left(\mathrm{IC}_{50}, \mu \mathrm{g} / \mathrm{mL}\right)$ & & & & & & & Trolox \\
\hline TBARS formation inhibition & $33 \pm 3^{\mathrm{f}}$ & $92.0 \pm 0.7^{\mathrm{e}}$ & $97 \pm 3^{\mathrm{d}}$ & $23 \pm 1^{g}$ & $387 \pm 2^{\mathrm{a}}$ & $341 \pm 1^{b}$ & $9.1 \pm 0.3$ \\
\hline OxHLIA, $\Delta t=60 \mathrm{~min}$ & $122 \pm 8^{c}$ & $180 \pm 11^{b}$ & $180 \pm 9^{b}$ & $110 \pm 4^{\mathrm{c}}$ & n.a. & $175 \pm 13^{\mathrm{b}}$ & $21.2 \pm 0.7$ \\
\hline OxHLIA, $\Delta t=120 \mathrm{~min}$ & $272 \pm 16^{\mathrm{c}}$ & $399 \pm 16^{b}$ & $374 \pm 3^{b}$ & $277 \pm 9^{c}$ & n.a. & $290 \pm 4^{c}$ & $41.1 \pm 0.8$ \\
\hline Cytotoxic activity $\left(\mathrm{GI}_{50}, \mu \mathrm{g} / \mathrm{mL}\right)$ & & & & & & & Ellipticine \\
\hline MCF-7 (breast adenocarcinoma) & $193 \pm 5^{c}$ & $>400$ & $>400$ & $227 \pm 19^{b}$ & $308 \pm 34^{\mathrm{a}}$ & $>400$ & $1.21 \pm 0.02$ \\
\hline NCI-H460 (lung carcinoma) & $69 \pm 3^{c}$ & $>400$ & $>400$ & $149 \pm 8^{b}$ & $199 \pm 7^{\mathrm{a}}$ & $>400$ & $0.9 \pm 0.1$ \\
\hline HeLa (cervical adenocarcinoma) & $91 \pm 5^{c}$ & $>400$ & $304 \pm 20^{c}$ & $314 \pm 8^{b}$ & $150 \pm 9^{d}$ & $329 \pm 4^{\mathrm{a}}$ & $1.03 \pm 0.09$ \\
\hline HepG2 (hepatocellular carcinoma) & $75 \pm 5^{c}$ & $>400$ & $264 \pm 7^{\mathrm{a}}$ & $>400$ & $73 \pm 5^{c}$ & $230 \pm 3^{b}$ & $1.10 \pm 0.09$ \\
\hline PLP2 (porcine liver primary cells) & $268 \pm 12^{*}$ & $>400$ & $>400$ & $>400$ & $252 \pm 9^{*}$ & $>400$ & $2.3 \pm 0.2$ \\
\hline Anti-inflammatory activity $\left(\mathrm{IC}_{50}, \mu \mathrm{g} / \mathrm{mL}\right)$ & & & & & & & Dexamethasone \\
\hline RAW 246.7 (murine macrophage cells) & $183 \pm 10^{\mathrm{c}}$ & $>400$ & $>400$ & $>400$ & $204 \pm 8^{b}$ & $291 \pm 8^{\mathrm{a}}$ & $16 \pm 1$ \\
\hline
\end{tabular}

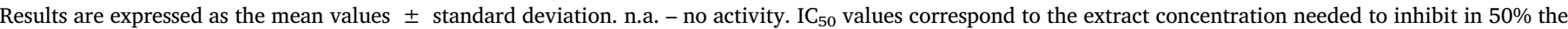

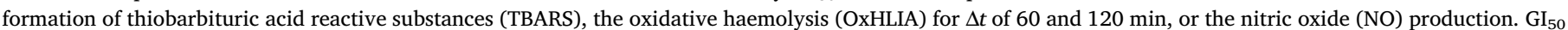

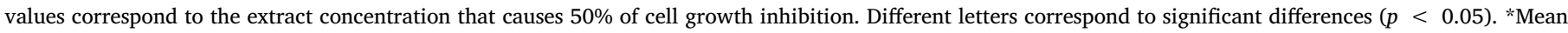
statistical differences obtained by Student's $t$-test.

(Petropoulos et al., 2018Petropoulos, Pereira, Ntatsi, et al., 2018; Petropoulos, Pereira, Tzortzakis, et al., 2018). The obtained results are in agreement with the literature reports. The antioxidant potential differences in head samples collected at different maturation stages have already been verified in other species. For instance, Chahdoura et al. (2014) reported that the vegetative stage of cactus plant (Opuntia spp.) flowers had higher antioxidant potential than full flowering and post flowering stages. Also, the immature peduncles of Hovenia dulcis presented higher antioxidant activity comparing to peduncles at advanced maturity stages (Morales et al., 2017). To the best of the authors' knowledge, this is the first study regarding the antioxidant activity of cardoon heads collected in different harvest stages and measuring their (antihaemolytic) activity by the OxHLIA assay.

The cytotoxic effects of cardoon heads extract are shown in Table 3. The results are presented as the concentration of the extracts that causes $50 \%$ inhibition in cell proliferation, where lower $\mathrm{GI}_{50}$ values represent a higher cytotoxic potential of the tested extracts. The extract obtained from the samples with lower maturity (Car A) revealed the highest cytotoxicity against the tumor cell lines tested. For HepG2 tumour cell line, the extract of sample Car E also revealed high activity, similar to that demonstrated by sample Car A, recording a $\mathrm{GI}_{50}$ of $73 \mu \mathrm{g} / \mathrm{mL}$. The remaining extracts (Car B, C, D, and F) showed no hepatotoxicity to the non-tumour PLP2 cells, with $\mathrm{GI}_{50}$ values $>400 \mu \mathrm{g}$ / $\mathrm{mL}$.

The influence of genotype on the cytotoxic properties of cardoon seeds has been already evaluated by Petropoulos, Fernandes, Pereira, et al. (2019), Petropoulos, Fernandes, Tzortzakis, et al. (2019) using the same cell lines tested herein, finding significant activities against the tumour cell lines, and no activity against the non-tumour cell line (PLP2) $\left(\mathrm{GI}_{50}>400 \mu \mathrm{g} / \mathrm{mL}\right)$. Mileo, Di Venere, Linsalata, Fraioli, and Miccadei (2012) also demonstrated the toxicity induced by methanolic extracts of cardoon heads against several types of breast cancer (BT549, MCF-7, MDA-MB231, and T47D), while Pagano et al. (2016) reported that artichoke by-products (bracts and leaves) exhibited significant cellular antioxidant activity through the inhibition of hydroperoxideinduced oxidative stress in HepG2 cells, which was attributed to the abundance in caffeoylquinic acids. The possible correlation of the high content in phenolic compounds and the anticancer activities demonstrated by cardoon extracts has also been pointed out by other authors (Petropoulos, Fernandes, Tzortzakis, et al., 2019).

The anti-inflammatory potential was tested through the measurement of the capacity of the extracts to inhibiting the NO production. NO is a proinflammatory mediator with an important role in the inflammatory process and several researches have been carried out to discover new substances that inhibit its production effectively (Aktan, 2004). The $\mathrm{IC}_{50}$ values $(\mu \mathrm{g} / \mathrm{mL})$ obtained regarding the anti- inflammatory potential of cardoon inflorescences are presented in Table 3. Only the immature (Car A) and senescent head samples (Car E and F) demonstrated a significant capacity to inhibit the NO production by the murine macrophage cell line (RAW 146.7), particularly Car A which exhibited the lowest IC $_{50}$ value $(183 \mu \mathrm{g} / \mathrm{mL})$. The rest of the samples (Car B, C, and D) did not reveal anti-inflammatory potential $\left(\mathrm{IC}_{50}>400 \mu \mathrm{g} / \mathrm{mL}\right)$. There are very few studies regarding the antiinflammatory potential of cardoon. Salem et al. (2017) evaluated the in vivo anti-inflammatory activity of artichoke (C. cardunculus var. scolymus) extracts through the Carr test, suggesting that the anti-inflammatory activity is caused by a synergistic action of the phenolic compounds present in the obtained ethanolic extracts. To the best of authors' knowledge, this is the first study reporting the anti-inflammatory potential of cardoon heads collected at different harvesting times assayed through this cell-based method.

The antimicrobial activities of the studied samples are presented in Table 4. All the extracts revealed bacteriostatic and bactericidal activity, but none of them demonstrated higher or similar activity than the positive controls used, namely streptomycin and ampicillin. In general, the lowest MIC values were obtained for the sample Car E, except for the case of Bacillus cereus where Car D showed the highest efficiency (MIC $=0.38 \mathrm{mg} / \mathrm{mL}$ ) (Table 4). Moreover, the assayed Gram-positive bacteria presented higher susceptibility than the Gramnegative ones. This fact is in agreement with former reports, which described that the membrane characteristics of Gram-positive bacteria led to a higher vulnerability against the plant extracts (Gyawali, Hayek, \& Ibrahim, 2014). In general, the MIC and MBC values were lower than those described by Dias et al. (2018) for hydroethanolic extracts of cardoon inflorescences of different genotypes; however, the bacteria strains used in that study were clinical isolates with a strong resistance profile against antibiotics, whereas in the present study they were mainly ATCC strains, with no resistance profile against antibiotics.

The antifungal potential of the cardoon heads against Aspergillus spp. and Penicillium spp. was also assessed. As presented in Table 4, the tested extracts possessed higher MIC and MFC values than the positive controls, namely ketoconazole and bifonazole, with the extract Car D exhibiting the lowest MIC and MFC values for all the fungi species tested. The remaining extracts showed a variable activity depending on the fungi species. In general, the extracts Car B and Car D were the ones that revealed the lowest potential for all the fungi species, except against $A$. niger, where the extract from sample Car A was the one that revealed the lowest activity, with the highest MIC value $(9.32 \mathrm{mg} / \mathrm{mL})$.

Other reports make reference to the high antimicrobial potential of different cardoon tissues (namely flowers, heads, leaves, and rhizomes). In particular, Falleh et al. (2008) and Scavo et al. (2019) described significant antimicrobial activity of plant tissues against different 
Table 4

Antibacterial and antifungal activities of Cynara cardunculus var. altilis head extracts.

\begin{tabular}{|c|c|c|c|c|c|c|c|c|c|c|c|c|c|c|c|c|}
\hline \multirow[b]{2}{*}{ Antibacterial activity $(\mathrm{mg} / \mathrm{mL})$} & \multicolumn{2}{|c|}{ Car A } & \multicolumn{2}{|c|}{ Car B } & \multicolumn{2}{|c|}{ Car C } & \multicolumn{2}{|l|}{ Car D } & \multicolumn{2}{|c|}{ Car E } & \multicolumn{2}{|c|}{ Car F } & \multicolumn{2}{|c|}{ Streptomycin } & \multicolumn{2}{|c|}{ Ampicillin } \\
\hline & MIC & MBC & MIC & MBC & MIC & MBC & MIC & MBC & MIC & MBC & MIC & MBC & MIC & MBC & MIC & MBC \\
\hline Bacillus cereus & 1.75 & 3.49 & 1.53 & 3.06 & 0.75 & 1.51 & 0.38 & 0.77 & 0.59 & 1.18 & 0.59 & 1.17 & 0.10 & 0.20 & 0.25 & 0.40 \\
\hline Staphylococcus aureus & 3.49 & 6.99 & 3.06 & 6.12 & 3.01 & 6.03 & 1.54 & 3.08 & 0.59 & 1.18 & 1.17 & 2.34 & 0.04 & 0.10 & 0.25 & 0.45 \\
\hline Listeria monocytogenes & 3.49 & 6.99 & 3.06 & 6.12 & 3.01 & 6.03 & 1.54 & 3.07 & 1.18 & 2.35 & 1.17 & 2.34 & 0.20 & 0.30 & 0.40 & 0.50 \\
\hline Enterobacter cloacae & 3.49 & 6.99 & 3.06 & 6.12 & 3.01 & 6.03 & 1.54 & 3.07 & 0.59 & 1.18 & 1.17 & 2.34 & 0.20 & 0.30 & 0.25 & 0.50 \\
\hline Escherichia coli & 1.75 & 3.49 & 1.53 & 3.06 & 3.01 & 6.03 & 3.075 & 6.15 & 1.18 & 2.35 & 2.34 & 4.69 & 0.20 & 0.30 & 0.40 & 0.50 \\
\hline \multirow{2}{*}{ Salmonella typhimurium } & 3.49 & 6.99 & 3.06 & 6.12 & 3.01 & 6.03 & 1.54 & 3.07 & 0.59 & 1.18 & 2.34 & 4.69 & 0.20 & 0.30 & 0.75 & 1.20 \\
\hline & & & & & & & & & & & & & \multicolumn{2}{|c|}{ Ketoconazole } & \multicolumn{2}{|c|}{ Bifonazole } \\
\hline Antifungal activity $(\mathrm{mg} / \mathrm{mL})$ & MIC & MFC & MIC & MFC & MIC & MFC & MIC & MFC & MIC & MFC & MIC & MFC & MIC & MFC & MIC & MFC \\
\hline Aspergillus fumigatus & 1.16 & 2.33 & 4.08 & 8.16 & 1.51 & 3.01 & 0.51 & 1.02 & 1.80 & 3.60 & 0.88 & 1.76 & 0.25 & 0.50 & 0.15 & 0.20 \\
\hline Aspergillus versicolor & 1.16 & 2.33 & 1.02 & 2.04 & 6.03 & $>6.03$ & 0.26 & 0.51 & 1.80 & 3.60 & 3.52 & 7.04 & 0.2 & 0.5 & 0.1 & 0.2 \\
\hline Aspergillus niger & 9.32 & $>9.32$ & 4.08 & 8.16 & 1.51 & 3.01 & 0.51 & 1.02 & 3.60 & 7.18 & 1.76 & 3.52 & 0.2 & 0.5 & 0.15 & 0.2 \\
\hline Penicillium funiculosum & 0.58 & 1.16 & 1.02 & 2.04 & 6.03 & $>6.03$ & 0.51 & 1.02 & 1.80 & 3.60 & 0.88 & 1.76 & 0.2 & 0.5 & 0.2 & 0.25 \\
\hline Penicillium ochrochloron & 0.58 & 1.16 & 1.02 & 2.04 & 1.51 & 3.01 & 0.51 & 1.02 & 0.90 & 1.80 & 0.88 & 1.76 & 1.0 & 1.5 & 0.2 & 0.25 \\
\hline Penicillium verrucosum var. cyclopium & 0.58 & 1.16 & 1.02 & 2.04 & 6.03 & $>6.03$ & 0.51 & 1.02 & 1.80 & 3.60 & 0.88 & 1.76 & 0.2 & 0.3 & 0.1 & 0.2 \\
\hline
\end{tabular}

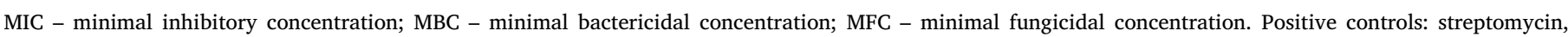
ampicillin, ketoconazole, and bifonazole.

bacteria (Bacillus cereus, Escherichia coli, Pseudomonas aeruginosa, Pseudomonas fluorescens, Salmonella enterica, and Staphylococcus aureus) and fungi (Aspergillus niger, Penicillium oxalicum, and Candida albicans) species.

Overall, the maturation stage of cardoon heads had a significant influence on the in vitro bioactivities. Thus, this information can be useful to improve the health benefits of cardoon heads and could be a means to take full advantage of its potential and to increase the added value of the crop. However, further studies are needed to correlate the identified compounds with the observed bioactivities and to reveal the mechanisms of action, as well as to optimize the extraction process for the isolation of these compounds. Finally, the study of the influence of the maturation stage on the remaining plant tissues of cardoon would be extremely useful.

\section{Conclusion}

The cardoon heads, usually considered as food, could have an added commercial value as a source of bioactive compounds. Caffeoylquinic acids and flavone glycosides (especially apigenin derivatives) were the most abundant classes of phenolic compounds in cardoon heads. The earliest maturation stages (Car A) stood out showing the highest content in polyphenols, to further decrease with the state of maturity. The immature heads also revealed higher cytotoxic and anti-inflammatory potential, with lower $\mathrm{GI}_{50}$ and $\mathrm{IC}_{50}$ values, respectively. Immature heads (Car A) also exhibited higher antioxidant potential in the cellbased assays, with lower $\mathrm{IC}_{50}$ values. All the extracts possessed significant antibacterial activity, with extracts from Car B showing the best results, with the lowest MIC and MBC values. Regarding the antifungal potential, most of the extracts presented inhibitory capacity against the tested fungi species. The sample of advanced maturation stage (Car E) was the one that revealed higher activity, with lower MIC and MFC values. This study allowed for a more complete characterization of the cardoon heads in terms of phenolic compounds composition and the effect of maturation stage on polyphenolic compounds profile and bioactivities. The presented results could be useful, since simple cultivation practices such as harvesting time may allow for a significant enhancement of the bioactive profile of cardoon heads, thus increasing the overall added value of the crop.

\section{CRediT authorship contribution statement}

Filipa Mandim: Methodology, Investigation, Writing - original draft. Spyridon A. Petropoulos: Methodology, Writing - review \& editing. Kyriakos D. Giannoulis: Methodology, Investigation. Maria
Inês Dias: Methodology, Writing - original draft. Ângela Fernandes: Methodology, Writing - original draft. José Pinela: Methodology. Marina Kostic: Methodology. Marina Soković: Methodology. Lillian Barros: Conceptualization, Methodology, Writing - review \& editing. Celestino Santos-Buelga: Conceptualization, Methodology, Writing review \& editing. Isabel C.F.R. Ferreira: Conceptualization, Methodology, Writing - review \& editing.

\section{Declaration of Competing Interest}

The authors declare that they have no known competing financial interests or personal relationships that could have appeared to influence the work reported in this paper.

\section{Acknowledgements}

The authors are grateful to the Foundation for Science and Technology (FCT, Portugal) for financial support through national funds FCT/Ministério da Ciência, Tecnologia e Ensino Superior (MCTES), Portugal to CIMO (UIDB/00690/2020) and the research contracts of L. Barros, M.I. Dias, A. Fernandes, and J. Pinela (national funding by FCT, through the institutional scientific employment program-contract), and F. Mandim PhD grant (SFRH/BD/146614/2019). The authors are also grateful to FEDER-Interreg España-Portugal programme for financial support through the project 0377_Iberphenol_6_E, to the project TRANSCoLAB (0612_TRANS_CO_LAB_2_P), and to the Ministry of Education, Science and Technological Development of the Republic of Serbia for Grant No. 173032.

\section{References}

Aktan, F. (2004). iNOS-mediated nitric oxide production and its regulation. Life Sciences, 75(6), 639-653.

Alghazeer, R., El-Saltani, H., Saleh, N. A., Al-Najjar, A., Naili, M. B., Hebail, F., \& El-Deeb, H. (2012). Antioxidant and antimicrobial activities of Cynara scolymus L. rhizomes. Modern Applied Science, 6(7), 54-63.

Archontoulis, S. V., Struik, P. C., Vos, J., \& Danalatos, N. G. (2013). Phenological growth stages of Cynara cardunculus: Codification and description according to the $\mathrm{BBCH}$ scale. Annals of Applied B, 156(2), 253-270.

Barros, L., Pereira, E., Calhelha, R. C., Dueñas, M., Carvalho, A. M., Santos-Buelga, C., \& Ferreira, I. C. F. R. (2013). Bioactivity and chemical characterization in hydrophilic and lipophilic compounds of Chenopodium ambrosioides L. Journal of Functional Foods, 5(4), 1732-1740.

Bessada, S. M. F., Barreira, J. C. M., Barros, L., Ferreira, I. C. F. R., \& Oliveira, M. B. P. P. (2016). Phenolic profile and antioxidant activity of Coleostephus myconis (L.) Rchb.f.: An underexploited and highly disseminated species. Industrial Crops and Products, 89, 45-51.

Chahdoura, H., Barreira, J. C. M., Barros, L., Santos-Buelga, C., Ferreira, I. C. F. R., \& Achour, L. (2014). Phytochemical characterization and antioxidant activity of 
Opuntia microdasys (Lehm.) Pfeiff flowers in different stages of maturity. Journal of Functional Foods, 9, 27-37.

Conceição, C., Martins, P., Alvarenga, N., Dias, J., Lamy, E., Garrido, L., Gomes, S., Freiras, S., Belo, A., Brás, T., Paulino, A., \& Duarte, M. F. (2016). Cynara cardunculus: Use in cheesemaking and pharmaceutical applications. In N. Koca (Ed.), Technological Approaches for Novel Applications in Dairy Processing (Vol. 1, pp. 73-107). IntechOpen.

Dias, M. I., Barros, L., Barreira, J. C. M., Alves, M. J., Barracosa, P., \& Ferreira, I. C. F. R. (2018). Phenolic profile and bioactivity of cardoon (Cynara cardunculus L.) inflorescence parts: Selecting the best genotype for food applications. Food Chemistry, 268, 196-202.

Falleh, H., Ksouri, R., Chaieb, K., Karray-Bouraoui, N., Trabelsi, N., Boulaaba, M., \& Abdelly, C. (2008). Phenolic composition of Cynara cardunculus L. organs, and their biological activities. Comptes Rendus - Biologies, 331(5), 372-379.

Ferreira, I. C. F. R., Martins, N., \& Barros, L. (2017). Phenolic compounds and its bioavailability: In vitro bioactive compounds or health promoters? Advances in Food and Nutrition Research, 82, 1-44.

Gominho, J., Curt, M. D., Lourenço, A., Fernández, J., \& Pereira, H. (2018). Cynara cardunculus L. as a biomass and multi-purpose crop: A review of 30 years of research. Biomass and Bioenergy, 109, 257-275.

Gostin, A. I., \& Waisundara, V. Y. (2019). Edible flowers as functional food: A review on artichoke (Cynara cardunculus L.). Trends in Food Science and Technology, 86, 381-391.

Gouveia, S. C., \& Castilho, P. C. (2012). Phenolic composition and antioxidant capacity of cultivated artichoke, Madeira cardoon and artichoke-based dietary supplements. Food Research International, 48(2), 712-724.

Gyawali, R., Hayek, S. A., \& Ibrahim, S. A. (2014). Plant extracts as antimicrobials in food products: Mechanisms of action, extraction methods, and applications. Handbook of Natural Antimicrobials for Food Safety and Quality (pp. 49-68). Elsevier Ltd.

Kollia, E., Markaki, P., Zoumpoulakis, P., \& Proestos, C. (2017). Antioxidant activity of Cynara scolymus L. and Cynara cardunculus L. extracts obtained by different extraction techniques. Natural Product Research, 31, 1163-1167.

Lombardo, S., Pandino, G., Mauromicale, G., Knödler, M., Carle, R., \& Schieber, A. (2010). Influence of genotype, harvest time and plant part on polyphenolic composition of globe artichoke [Cynara cardunculus L. var. scolymus (L.) Fiori]. Food Chemistry, 119(3), 1175-1181.

Mandim, F., Barros, L., Calhelha, R. C., Abreu, R. M. V., Pinela, J., Alves, M. J., ... Ferreira, I. C. F. R. (2019). Calluna vulgaris (L.) Hull: Chemical characterization, evaluation of its bioactive properties and effect on the vaginal microbiota. Food and Function, 10, 78-89.

Mileo, A. M., Di Venere, D., Linsalata, V., Fraioli, R., \& Miccadei, S. (2012). Artichoke polyphenols induce apoptosis and decrease the invasive potential of the human breast cancer cell line MDA-MB231. Journal of Cellular Physiology, 227(9), 3301-3309.

Morales, P., Maieves, H. A., Dias, M. I., Calhella, R. C., Sánchez-Mata, M. C., SantosBuelga, C., ... Ferreira, I. C. F. R. (2017). Hovenia dulcis Thunb. pseudofruits as functional foods: Phytochemicals and bioactive properties in different maturity stages. Journal of Functional Foods, 29, 37-45.

Pagano, I., Piccinelli, A. L., Celano, R., Campone, L., Gazzerro, P., Falco, E., \& Rastrelli, L. (2016). Chemical profile and cellular antioxidant activity of artichoke by-products. Food \& Function, 7(12), 4841-4850.

Pandino, G., Courts, F. L., Lombardo, S., Mauromicale, G., \& Williamson, G. (2010). Caffeoylquinic acids and flavonoids in the immature Inflorescence of globe artichoke, wild cardoon, and cultivated cardoon. Journal of Agricultural and Food Chemistry,
58(2), 1026-1031.

Pandino, G., Lombardo, S., Lo Monaco, A., \& Mauromicale, G. (2013). Choice of time of harvest influences the polyphenol profile of globe artichoke. Journal of Functional Foods, 5(4), 1822-1828.

Pandino, G., Lombardo, S., Williamson, G., \& Mauromicale, G. (2012). Polyphenol profile and content in wild and cultivated Cynara cardunculus L. Italian Journal of Agronomy, 7(3), 254-261.

Pereira, C., Barros, L., Carvalho, A. M., Santos-Buelga, C., \& Ferreira, I. C. F. R. (2015). Infusions of artichoke and milk thistle represent a good source of phenolic acids and flavonoids. Food and Function, 6(1), 56-62.

Petropoulos, S. A., Fernandes, Â., Pereira, C., Tzortzakis, N., Vaz, J., Soković, M., ... Ferreira, I. C. F. R. (2019). Bioactivities, chemical composition and nutritional value of Cynara cardunculus L. seeds. Food Chemistry, 289, 404-412.

Petropoulos, S. A., Fernandes, Â., Tzortzakis, N., Sokovic, M., Ciric, A., Barros, L., \& Ferreira, I. C. F. R. (2019). Bioactive compounds content and antimicrobial activities of wild edible Asteraceae species of the Mediterranean flora under commercial cultivation conditions. Food Research International, 119, 859-868.

Petropoulos, S. A., Pereira, C., Barros, L., \& Ferreira, I. C. F. R. (2017). Leaf parts from Greek artichoke genotypes as a good source of bioactive compounds and antioxidants. Food and Function, 8(5), 2022-2029.

Petropoulos, S. A., Pereira, C., Ntatsi, G., Danalatos, N., Barros, L., \& Ferreira, I. C. F. R. (2018). Nutritional value and chemical composition of Greek artichoke genotypes. Food Chemistry, 267, 296-302.

Petropoulos, S. A., Pereira, C., Tzortzakis, N., Barros, L., \& Ferreira, I. C. F. R. (2018). Nutritional value and bioactive compounds characterization of plant parts from Cynara cardunculus L. (Asteraceae) cultivated in central Greece. Frontiers Plant Science, 9, 459.

Piluzza, G., Molinu, M. G., Re, G. A., \& Sulas, L. (2019). Phenolic compounds content and antioxidant capacity in cardoon achenes from different head orders. Natural Product Research, 5, 1-5.

Ramos, P. A. B., Santos, S. A. O., Guerra, Â. R., Guerreiro, O., Freire, C. S. R., Rocha, S. M., ... Silvestre, A. J. D. (2014). Phenolic composition and antioxidant activity of different morphological parts of Cynara cardunculus L. var. altilis (DC). Industrial Crops and Products, 61, 460-471.

Russo, A., Perri, M., Cione, E., Di Gioia, M. L., Nardi, M., \& Cristina Caroleo, M. (2017). Biochemical and chemical characterization of Cynara cardunculus L. extract and its potential use as co-adjuvant therapy of chronic myeloid leukemia. Journal of Ethnopharmacology, 202, 184-191.

Salem, M. Ben, Affes, H., Athmouni, K., Ksouda, K., Dhouibi, R., Sahnoun, Z., ... Zeghal, K. M. (2017). Chemicals compositions, antioxidant and anti-Inflammatory activity of Cynara scolymus leaves extracts, and analysis of major bioactive polyphenols by HPLC. Evidence-Based Complementary and Alternative Medicine, 2017, 4951937.

Scavo, A., Pandino, G., Restuccia, C., Parafati, L., Cirvilleri, G., \& Mauromicale, G. (2019). Antimicrobial activity of cultivated cardoon (Cynara cardunculus L. var. altilis DC.) leaf extracts against bacterial species of agricultural and food interest. Industrial Crops and Products, 129, 206-211.

Souilem, F., Fernandes, Â., Calhelha, R. C., Barreira, J. C. M., Barros, L., Skhiri, F., ... Ferreira, I. C. F. R. (2017). Wild mushrooms and their mycelia as sources of bioactive compounds: Antioxidant, anti-inflammatory and cytotoxic properties. Food Chemistry, 230, 40-48.

Wahba, H. E., Sarhan, A. Z., Salama, A. B., Sharaf-Eldin, M. A., \& Gad, H. M. (2017). Effect of seasonal variation on the growth and chemical composition of Cynara cardunculus L. plants. Journal of Materials and Environmental. Science, 8(1), 318-323. 